\title{
ESTRUTURA POPULACIONAL DE Symphonia globulifera L. f. (CLUSIACEAE) EM FRAGMENTOS DE MATA ATLÂNTICA DE BAIXAdA PERIODICAMENTE ALAGADA ${ }^{1}$
}

\author{
Ezequiel Moraes dos Santos², Marcelo Trindade Nascimento ${ }^{3}$
}

(recebido: 16 de agosto de 2010; aceito: 22 de dezembro de 2011)

\begin{abstract}
RESUMO: Neste trabalho, avaliou-se a estrutura populacional de Symphonia globulifera em fragmentos florestais periodicamente alagados, nas Reservas Biológicas de Poço das Antas (RBPA) e União (RBU), Rio de Janeiro. Uma análise comparativa entre o papel das estratégias reprodutivas (via semente ou via vegetativa) na estrutura populacional foi também realizada. Para a amostragem, foram selecionadas três áreas na RBPA (PORT, CM e ARI) e uma área na RBU. Foram encontrados dois tipos de estrutura populacional para S. globulifera: 1) populações PORT e ARI com baixo recrutamento e predominância de indivíduos oriundos de sementes que podem estar em fase de senescência e 2) populações CM e RBU com grande número de recrutas oriundos de reprodução vegetativa. Plântulas e jovens apresentaram, em geral, um maior número de indivíduos de via vegetativa. Em relação aos adultos, ocorreu um predomínio de indivíduos oriundos de sementes. Esses padrões parecem estar relacionados ao regime hídrico de cada ambiente. A predominância nos pântanos de plântulas e jovens de via vegetativa e árvores oriundas de semente sugere a existência de uma forte mortalidade diferenciada nas fases de plântula e jovens de indivíduos oriundos de reprodução vegetativa.
\end{abstract}

Palavras-chave: Demografia, dinâmica populacional, fragmentação florestal, floresta alagada.

\section{POPULATION STRUCTURE OF Symphonia globulifera L. f. (CLUSIACEAE) IN FRAGMENTS OF SEASONALLY FLOODED LOWLAND ATLANTIC FOREST}

ABSTRACT: This paper assesses the population structure of Symphonia globulifera in forest fragments of lowland Atlantic Forest in the Poço das Antas Biological Reserve (RBPA) and the União Biological Reserve (RBU), Rio de Janeiro, Brazil. A comparative analysis of the role of seed and vegetative reproduction in the plant population structure was also carried out. Three sampling areas were selected in the RBPA (PORT, CM and ARI) and one area in the RBU. Two types of population structure were found: 1) populations with low recruitment and with several individuals originated from seeds that appeared to be senescent (PORT and ARI), and 2) populations with high number of recruits from vegetative reproduction (CM and RBU). Seedlings and saplings showed, in general, a higher number of individuals from vegetative reproduction. On the other hand, adults had a predominance of individuals from seed reproduction. These structured patterns appear to be related to the water regimes in each area. Therefore, these data suggest the occurrence of a strong differentiated mortality of seedlings and saplings from vegetative reproduction.

Key words: Demography, population dynamics, forest fragmentation, swamp forest.

\section{INTRODUÇÃO}

O Brasil possui cerca de $2 \%$ de terras úmidas. Estas têm sido pouco estudadas do ponto de vista da ecologia vegetal. Os trabalhos existentes referem-se às florestas inundáveis da Amazônia (PAROLIN, 2009) e do Pantanal Mato-grossense, a maior planície de inundação do mundo (NASCIMENTO; CUNHA, 1989; PRANCE; SCHALLER, 1982). Para a Mata Atlântica, a situação é ainda mais crítica e Scarano et al. (1997) fazem menção da importância de pesquisas serem direcionadas às florestas inundáveis na Mata Atlântica, visto que restam menos de $2 \%$ da área original desta fitofisionomia.
Estudos detalhados a respeito da dinâmica de população de espécies tropicais são comuns (CORRÊA; BERG, 2002; NASCIMENTO; PROCTOR, 1997). Entretanto, poucos estudos enfocaram espécies de florestas periodicamente alagadas (MARQUES; JOLY, 2000; SOUZA et al., 2007).

Nas plantas, o sistema de reprodução afeta fortemente a estrutura ecológica de populações (HOSSAERT-MCKEY et al., 1996). Um número relativo de espécies de plantas possui reprodução tanto por via sexual quanto por via vegetativa (brotamento). A via sexual envolve a produção de sementes e, consequentemente, o desenvolvimento de plântulas e a via vegetativa o

${ }^{1}$ Parte da dissertação de mestrado do primeiro autor.

${ }^{2}$ Biólogo, Pós-graduando em Biociências e Biotecnologia - Centro de Biociências e Biotecnologia - Universidade Estadual do Norte Fluminense Av. Alberto Lamego 2000 - 28013-620 - Campos dos Goytacazes, RJ, Brasil - ezeqmoraes@terra.com.br

${ }^{3}$ Biólogo, Professor Dr. em Ecologia Vegetal - Laboratório de Ciências Ambientais - Centro de Biociência e Biotecnologia - Universidade Estadual do Norte Fluminense - Av. Alberto Lamego 2000 - 28013-620 - Campos dos Goytacazes, RJ, Brasil - mtn@uenf.br

Cerne, Lavras, v. 18, n. 2, p. 265-273, abr./jun. 2012 
crescimento clonal, gerando indivíduos conectados ou não (BELLINGHAM; SPARROW, 2000). Essas estratégias reprodutivas combinadas podem produzir considerável variação entre populações em resposta a fatores ecológicos (SILANDER JUNIOR, 1985). Em geral, a frequência relativa de brotamento é maior em comunidades sujeitas a perturbação (BELLINGHAM; SPARROW, 2000). Ambientes inundados ou periodicamente inundados, comumente apresentam espécies de plantas com características de alternar o modo reprodutivo de sexual para vegetativo ou vice-versa, (CRAWFORD, 1992; SCARANO et al., 1997). Em alguns casos, determinadas espécies podem manter ambos, com ajuste da dispersão de sementes em época favorável, ou seja, o período das cheias.

Entre as espécies arbóreas tropicais adaptadas às áreas periodicamente inundadas, destaca-se a Symphonia globulifera, uma Clusiaceae conhecida por guanandi ou anani. Essa espécie possui distribuição natural através de três continentes, atingindo latitudes que vão desde $20^{\circ} \mathrm{N}$ (sul do México) a $20^{\circ} \mathrm{S}$ (Brasil-São Paulo), espalhando-se pelo oeste africano e indiano (GILL et al., 1998). No Brasil, está distribuída pelas matas de várzea úmida na região amazônica e em terrenos alagadiços da mata pluvial atlântica (pântanos), em geral formando densamentos quase puros. Essa espécie domina o dossel e apresenta propagação por sementes e por via vegetativa por meio de brotamentos das raízes (SCARANO, 2006; SCARANO et al., 1997). Suas flores e frutos são considerados um importante recurso alimentar para a fauna (ALDRICH; HAMRICK, 1998; BITTRICH; AMARAL, 1996; GILL et al., 1998). Peres (1986) demonstrou que a espécie é única na provisão de néctar para o mico-leão-dourado (Leontopithecus rosalia (Linnaeus, 1766)), tendo a mesma contribuído em 51\% da dieta desse primata entre os meses de maio a agosto. Quanto à estrutura da população, não se conhece qualquer estudo para S. globulifera na Mata Atlântica. Os estudos ecológicos específicos já realizados estão relacionados à biologia reprodutiva (BITTRICH; AMARAL, 1996; GILL et al., 1998) e genética populacional (ALDRICH; HAMRICK, 1998) em áreas florestais da Amazônia e Costa Rica.

Neste trabalho, objetivou-se caracterizar a estrutura populacional de S. globulifera em fragmentos florestais de Mata Atlântica periodicamente inundada, nas Reservas Biológicas de Poço das Antas (RBPA) e União (RBU), enfatizando-se suas características de propagação por sementes versus vegetativa e testar a hipótese de que a estrutura das populações de $S$. globulifera nas matas de baixadas periodicamente inundadas é predominantemente composta por indivíduos de origem vegetativa face ao ambiente estressante e dinâmico que é o pântano.

\section{MATERIAL E MÉTODOS}

Este trabalho foi realizado em duas áreas de Mata Atlântica de baixada no Estado do Rio de Janeiro, estando a primeira localizada na Reserva Biológica de Poço das Antas (RBPA), município de Silva Jardim (22 $33^{\circ}$ ' 33' S) e ( $42^{\circ} 18$ ' 19" W) e a segunda na Reserva Biológica União (RBU), distrito de Rocha Leão, município de Rio das Ostras $\left(22^{\circ} 27^{\prime} 30^{\prime \prime} \mathrm{S}\right.$ e $\left.42^{\circ} 02^{\prime} 15^{\prime \prime} \mathrm{W}\right)$. Essas reservas encontram-se distantes entre si, cerca de $22 \mathrm{~km}$ em linha reta. Predomina, na região, o clima tropical chuvoso com estação seca no inverno (As) de Köppen. Segundo a classificação de Veloso et al. (1991), essa região está inserida no domínio da Floresta Ombrófila Densa de Terras Baixas. Sua flora arbórea é caracterizada pela presença de espécies típicas de áreas alagadas, tais como: Tabebuia cassinoides, Inga laurina, Calophyllum brasiliense e Symphonia globulifera (CARVALHO et al., 2006; LIMA et al., 2006). Maiores detalhes sobre o clima, solo e a vegetação da RBPA pode ser encontrada em Lima et al. (2006).

Quatro remanescentes de Floresta Ombrófila Densa de Terras baixas periodicamente inundadas (pântanos) foram selecionados, sendo três na RBPA (Portuense- PORT, Casa dos Morcegos- CM e AristidesARI) e um na RBU. A profundidade da lâmina d'agua em cada pântano foi mensurada em 20 pontos aleatórios (10 na borda e 10 no interior). Na RBPA, foi mensurada em duas ocasiões, em janeiro/99 após um período de 10 dias de estiagem e em março/99 após dez dias de precipitação (144,4 mm). O pântano da RBU foi mensurado em abril de1999. Por não ter sido amostrado no mesmo período, o pântano da RBU não foi comparado estatisticamente aos demais pântanos.

Em cada pântano foram alocadas aleatoriamente três parcelas de $50 \mathrm{~m} \times 20 \mathrm{~m}$. O total de 12 parcelas constituiu uma área amostral de 1,2 ha. Em cada parcela, foram marcados e medidos todos os indivíduos de $S$. globulifera com DAP $\geq 10 \mathrm{~cm}$ (diâmetro a altura do peito), sendo estes considerados adultos. Cinco subparcelas de $5 \mathrm{~m}$ x $5 \mathrm{~m}$ foram alocadas aleatoriamente dentro das parcelas, totalizando sessenta subparcelas onde foram etiquetados, medidos e mapeados todos os indivíduos $<10 \mathrm{~cm}$ de DAP

Cerne, Lavras, v. 18, n. 2, p. 265-273, abr./jun. 2012 
$\mathrm{e} \geq 50 \mathrm{~cm}$ altura (jovens) e $<50 \mathrm{~cm}$ de altura (plântulas). As coletas dos dados ocorreram nos meses de junho e julho de 1997, sendo registrados todos os indivíduos nas parcelas. Em maio de 1998, realizou-se uma nova excursão ao campo, onde todos os indivíduos jovens e plântulas registrados anteriormente foram remensurados e os novos indivíduos dessas classes foram medidos, etiquetados e mapeados. Os parâmetros amostrados foram: diâmetro à altura do peito (DAP a 1,30 $\mathrm{m}$ do solo), no caso de adultos; altura em metros e tipo de origem, por sementes (S) ou vegetativa (V). Foram considerados indivíduos oriundos da forma vegetativa, aqueles que apresentaram conexão ou vestígios de conexão visível entre raízes com a planta que lhes deu origem, enquanto que indivíduos de origem por sementes apresentam raízes pivotantes (SANTOS, 1999). Ainda, dependendo do estágio de desenvolvimento, pôde-se ver o cotilédone próximo à superfície do solo. O rastreamento foi feito por meio do prolongamento das raízes. Para a determinação do tipo reprodutivo dos indivíduos adultos, foi observado se a planta apresentava junto a sua base conectividade com outro indivíduo, sendo estes considerados de origem vegetativa. Entretanto, a população de clones adulta pode ter sido subestimada, uma vez que não foi possível aferir como e quando os indivíduos se separam uns dos outros, tornando-se independentes.

Na segunda semana de março de 1998, período em que a grande maioria dos frutos já havia caído, foi avaliado o número de sementes de $S$. globulifera presentes nas subparcelas de $5 \mathrm{~m} \times 5 \mathrm{~m}$ nas quatro áreas.

O padrão de distribuição espacial foi determinado utilizando-se o Índice de Dispersão de Morisita (BROWER; ZAR, 1977). Para a análise dos dados referentes ao crescimento da altura e crescimento relativo (ou seja, o percentual de crescimento do indivíduo em relação ao seu tamanho a partir do primeiro censo) da população de jovens e plântulas, somente os indivíduos sobreviventes da primeira amostragem e com altura inferior a 2,5 m foram considerados. Para a comparação das taxas de crescimento entre os tipos reprodutivos das populações de plântulas e jovens, foi utilizado teste estatístico não paramétrico de Mann-Whitney. O teste de Kruskal-Wallis foi utilizado para avaliar as diferenças entre as profundidades da lâmina d'agua dos pântanos. Para testar a diferença de mortalidade entre os tipos reprodutivos foi utilizado qui-quadrado. Todos os testes foram executados no programa StatView ${ }^{\circledR}$ (ABACUS CONCEPTS, 1995) para Macintosh.

\section{RESULTADOS}

Os dados referentes à altura de inundação mostraram que os quatro pântanos são distintos quanto ao regime de inundação, diferindo em relação à profundidade média da lâmina d'agua (Figura 1). Observações de campo indicaram também diferenças quanto ao tempo e ao tipo de escoamento e drenagem dos pântanos. PORT e ARI apresentaram características de pântanos mais profundos e com maior tempo de retenção de água, enquanto $\mathrm{CM}$ teve o menor valor médio e menor tempo de inundação $(\mathrm{p}=0,04)$. CM demonstrou ser o mais homogêneo apresentando menores valores de desvio padrão, enquanto o mais heterogêneo dos quatro pântanos estudados quanto a profundidade da lâmina d'agua foi o da RBU. Esse pântano apresentou a maior amplitude de valores, ocorrendo valores tão baixo como zero e tão altos como $52,3 \mathrm{~cm}$ de profundidade.

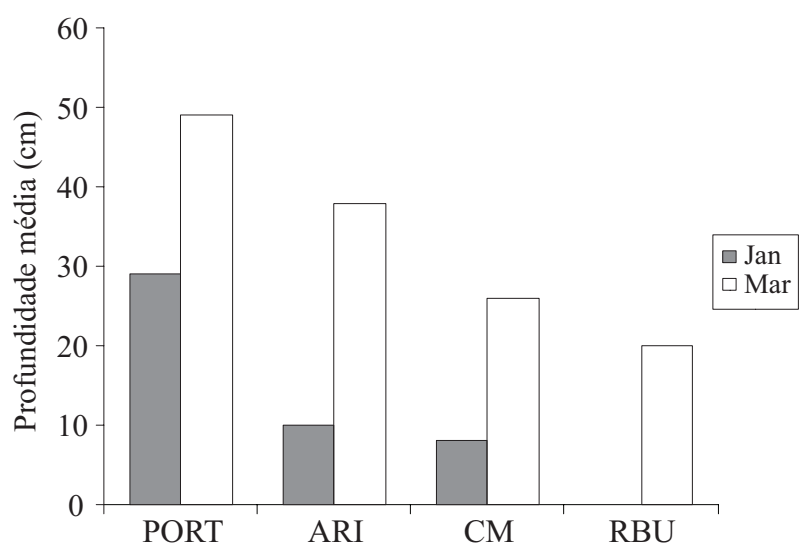

Figura 1 - Profundidade média $(\mathrm{cm})$ da lâmina d'água $(\mathrm{n}=10)$ em borda e interior de pântanos na RBPA(PORT, CM e ARI) medida em janeiro e março de 1999 e na RBU medida em abril 1999.

Figure 1 -Average depth (cm) of water column $(n=10)$ in edge and interior of swamp forests in the RBPA (PORT, CM and ARI) measured in January and March 1999 and in the RBU measured in April 1999.

Com base nas observações de campo quanto ao tempo e ao tipo de escoamento e, com os valores médios de profundidade encontrados, optou-se em separar os pântanos em dois grupos, os fundos (PORT e ARI) e os rasos (CM e RBU).

Um total de 431 indivíduos foi amostrado (1976 ind.ha ${ }^{-1}$ ) nas quatro áreas, distribuídos em 129 plântulas (860 ind.ha ${ }^{-1}$, sendo 633 ind.ha ${ }^{-1}$ de via vegetativa), 148 jovens (987 ind.ha ${ }^{-1}$, sendo 320 ind.ha $^{-1}$ de via vegetativa)

Cerne, Lavras, v. 18, n. 2, p. 265-273, abr./jun. 2012 
e 154 adultos (128 ind.ha ${ }^{-1}$, sendo 28 de via vegetativa). As populações CM e RBU (pântanos fundos) se diferenciaram de PORT e ARI (pântanos rasos) quanto à densidade de indivíduos, apresentando maior número de indivíduos em todos estádios de desenvolvimento (Figura 2). Em todas as quatro áreas, as plântulas apresentaram um maior número de indivíduos de via vegetativa. Em relação aos adultos, as quatro áreas tiveram predomínio de indivíduos oriundos de sementes. A população CM difere-se das demais, exceto da RBU, por apresentar um número muito superior de plântulas e jovens por área (Tabela 1), sendo estes, na sua maioria, de origem por sementes. Considerando-se todos os indivíduos amostrados em cada população, houve um predomínio de indivíduos de origem por semente, com exceção da população RBU, que apresentou um alto número de plântulas de origem vegetativa.

Segundo o Índice de Dispersão de Morisita, os adultos apresentaram uma distribuição aleatória, com valores variando entre 0,99 e 1,04. Jovens e plântulas apresentaram uma distribuição agregada independente da população estudada, com valores sempre superiores a 1,9.

Após 10 meses, maioria das plântulas de origem vegetativa $(43 \%)$ apresentou um percentual de crescimento de até $20 \%$ em relação ao seu tamanho da primeira medição. No entanto, foram observados alguns indivíduos com crescimento superior a $100 \%$ e outros com decréscimo de altura. Essas plântulas com decréscimo de altura corresponderam a $18 \%$ do total de sobreviventes de origem vegetativa. Para as plântulas de origem por sementes, foi observado um crescimento de $10 \%$ em relação ao tamanho da primeira medição, não sendo registrado nenhum indivíduo com decréscimo de altura e apenas um indivíduo com crescimento zero. Quando comparadas quanto à altura média, as plântulas de origem por sementes apresentaram um valor superior em $46 \%$, ou seja, $12 \mathrm{~cm}$ mais altas que as de origem vegetativa, com diferença significativa (Mann-Whitney U=960,5, p=0,0005). Embora as plântulas de origem vegetativa tenham crescido em média $23 \%$, enquanto que as de origem por sementes cresceram $16 \%$ em 10 meses, a diferença entre as alturas permaneceu em torno de $12 \mathrm{~cm}$, sendo esta significativa (Mann-Whitney $\mathrm{U}=971, \mathrm{p}=0,0003)$. No intervalo de 10 meses, $40 \%$ dos jovens cresceram até $10 \%$ em relação ao seu tamanho da primeira medição (julho/97). Entretanto, foram observados indivíduos que apresentaram decréscimo de altura $(15 \%$ para jovens de origem vegetativa e $20 \%$ para jovens de origem por sementes), ou seja, no segundo censo possuíam altura inferior ao primeiro, em geral em função de danos físicos por queda de galhos ou ataques de herbívoros.
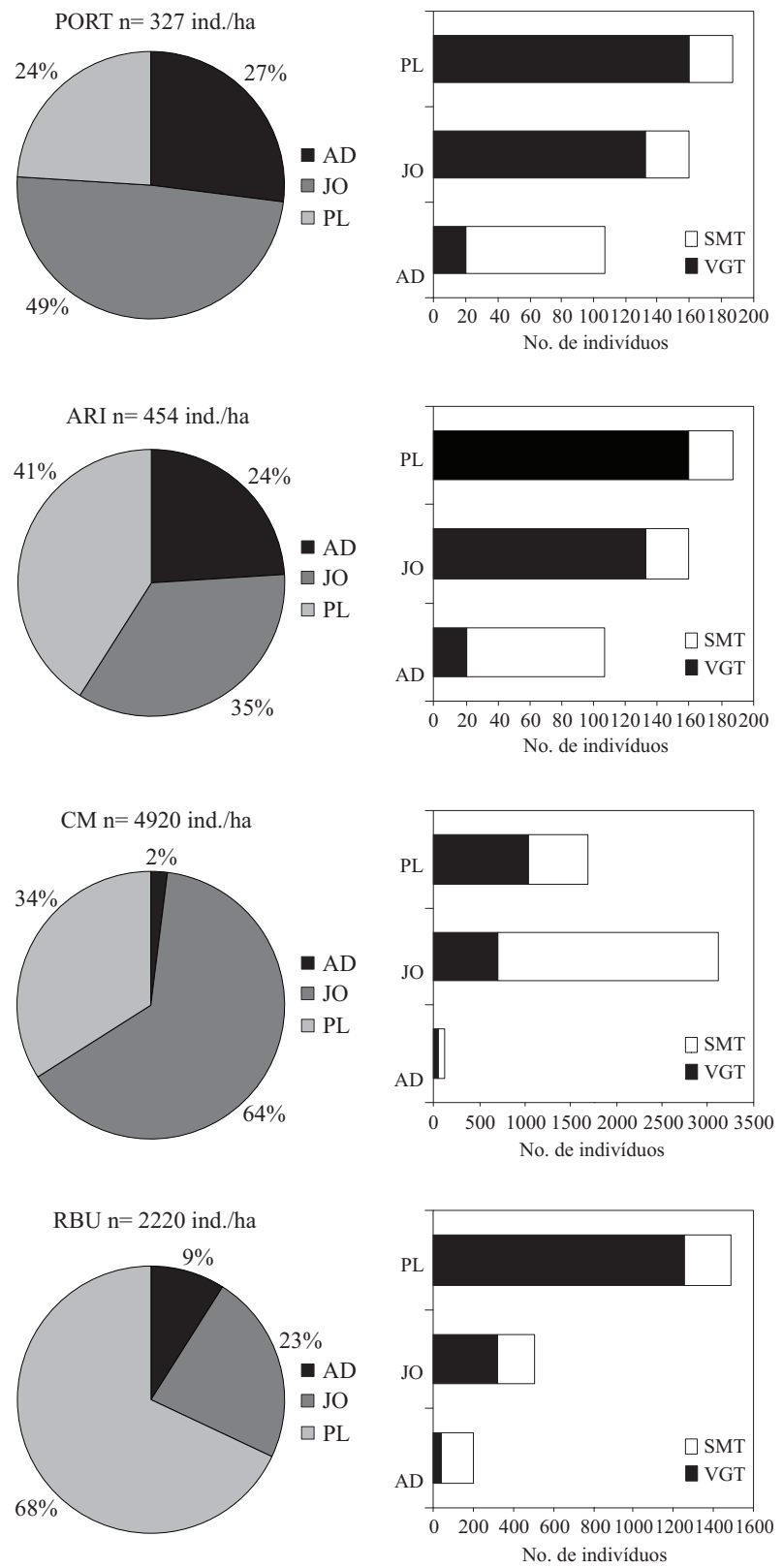

Figura 2 - Estrutura populacional de Symphonia globulifera em três pântanos da RBPA (PORT, ARI e CM) e um pântano na RBU. Distribuição ( $\mathrm{n}^{\mathrm{o}}$ ind.ha $\left.\mathrm{h}^{-1}\right)$ por idade com seus respectivos percentuais de ocorrência e por tipo reprodutivo, adultos (AD), jovens (JO), plântulas (PL), semente (SMT) e vegetativa (VGT).

Figure 2 - Population structure of Symphonia globulifera in swamp forests in the RBPA (PORT, ARI and CM) and in the RBU. Distribution $\left(n^{-}\right.$ind. $\left.h a^{-1}\right)$ by age with their respective percentages of occurrence and reproductive type, adults (AD), young (JO), seedling (PL), seed (SMT) and vegetative (VGT).

Cerne, Lavras, v. 18, n. 2, p. 265-273, abr./jun. 2012 
Tabela 1 - Número médio ( \pm desvio padrão) de plântulas (PL) e jovens (JO) por sub-parcelas de $25 \mathrm{~m}^{2}$ e adultos (AD) por $1.000 \mathrm{~m}^{2}$ de Symphonia globulifera em pântanos na RBPA (PORT, CM e ARI) e na RBU.

Table 1-Average number $( \pm S D)$ of seedlings $(P L)$ and saplings (JO) per $25 \mathrm{~m}^{2}$ and adults (AD) per 1,000 $\mathrm{m}^{2}$ of Symphonia globulifera in swamp forests in the RBPA (PORT, CM and ARI) and in the $R B U$.

\begin{tabular}{cccc}
\hline PORT & PL & JO & AD \\
\hline 1 & 0,0 & $0,8 \pm 1,8$ & $8,7 \pm 3,6$ \\
2 & 0,0 & 0,0 & \\
3 & $0,6 \pm 1,34$ & 0,0 & \\
\hline CM & & & \\
\hline 1 & $2,4 \pm 2,3$ & $6,8 \pm 5,9$ & $12,0 \pm 3,0$ \\
2 & $5,8 \pm 6,6$ & $9,2 \pm 6,7$ & \\
3 & $4,4 \pm 5,9$ & $7,4 \pm 11,7$ & \\
\hline ARI & & & \\
\hline 1 & $0,2 \pm 0,4$ & $0,2 \pm 0,4$ & $10,7 \pm 4,0$ \\
2 & $0,8 \pm 1,1$ & $1,0 \pm 1,0$ & \\
3 & $0,4 \pm 0,9$ & $0,2 \pm 0,4$ & \\
\hline RBU & & & \\
\hline 1 & $9,6 \pm 17,8$ & $1,2 \pm 0,4$ & $20,0 \pm 6,5$ \\
2 & 0,0 & 0,0 & \\
3 & $1,6 \pm 2,1$ & $2,6 \pm 2,9$ & \\
\hline
\end{tabular}

Em média, a população de jovens cresceu pouco no período de 10 meses, com os indivíduos de origem vegetativa apresentando crescimento médio de $1,2 \%$, enquanto os de sementes apresentaram um crescimento médio de $4 \%$. Os jovens de origem por sementes $(n=74)$ foram em média $16 \%$ mais altos que os indivíduos de origem vegetativa $(\mathrm{n}=43)$, porém essa diferença não foi significativa (MannWhitney $\mathrm{U}=1861,0 \mathrm{p}=0,127)$. No segundo censo, a diferença aumentou para $20 \%$, sendo significativa (MannWhitney $U=1976,5, p=0,0293$ ).

Em relação à mortalidade, foi observado que as plântulas tiveram uma mortalidade de $28 \%$, ou seja, 36 plântulas de um total de 129 plântulas amostradas, não havendo praticamente diferença entre as proporções por tipo reprodutivo (15\% para os indivíduos de origem vegetativa e $13 \%$ para os de origem por sementes). No entanto, quando comparada à mortalidade dentro do total de plântulas registradas por tipo reprodutivo ( $\mathrm{n}=96$ por sementes e $n=33$ por via vegetativa), a diferença se acentua, sendo estatisticamente significante $\left(\chi^{2}=12,3\right.$, g.l. $=1, p=0,0005)$. A mortalidade de plântulas de origem vegetativa foi de $20 \%(\mathrm{n}=19)$, enquanto que para as plântulas de origem por sementes o valor foi de $50 \%$ $(\mathrm{n}=17)$. A maioria das plântulas de origem vegetativa morreu antes de atingir $30 \mathrm{~cm}(74 \%)$, enquanto que a maioria das plântulas de origem por sementes (82\%) morreu quando possuiam altura entre 30 e $50 \mathrm{~cm}$. Dos 148 jovens amostrados $14 \%(\mathrm{n}=21)$ não sobreviveram após 10 meses. Não foi observada diferença significativa de mortalidade entre os tipos reprodutivos $\left(\chi^{2}=0,8\right.$, g.l. $=1$, $\mathrm{p}=0,36)$. A maioria dos jovens mortos de origem por sementes possuia altura entre 50 e $70 \mathrm{~cm}$, já para os de origem vegetativa ocorrreu uma maior mortalidade na classe de altura entre 90 e $110 \mathrm{~cm}$.

Um total de 318 sementes foi amostrado nos quatro pântanos. A maioria (60\%) destas já se encontrava em processo de germinação. A população PORT foi a que apresentou o menor número de sementes $/ \mathrm{m}^{2}(0,05)$, contrastando fortemente com as outras, 0,31 para $\mathrm{CM}$, 0,26 para ARI e 0,23 para RBU. Um total de 65 recrutas (novas plântulas) foi amostrado. A população $\mathrm{CM}$ foi a que apresentou o maior número de indivíduos novos de origem por semente, enquanto as outras populações apresentaram um maior número de indivíduos de origem vegetativa. As plântulas novas de origem por sementes $(\mathrm{n}=37)$ apresentaram estatura (altura média de $29 \mathrm{~cm})$ superior às de origem vegetativa $(\mathrm{n}=28)$ (altura média de $20 \mathrm{~cm}$ ), sendo essa diferença significativa estatisticamente (Mann-Whitney $\mathrm{U}=722,5, \mathrm{P}=0,0067$ ). O menor indivíduo amostrado apresentou $10 \mathrm{~cm}$ e o maior $48 \mathrm{~cm}$ de altura. Esses novos indivíduos significaram um incremento de $23 \%$ em toda população de plântulas de S. globulifera amostrada no período de julho de 1997 a maio de 1998. Analisando separadamente as populações, a população ARI foi a que apresentou maior percentual de incremento com $216 \%$, seguido das populações PORT (167\%), RBU $(16 \%)$ e CM (6\%).

Relacionando o estabelecimento de novas plântulas de origem por sementes com a amostragem de sementes aparentemente viáveis em cada população, a população $\mathrm{CM}$ foi a que apresentou maior percentual de estabelecimento (Tabela 2), seguida da população PORT. No entanto, o número de sementes amostrado nessa população foi extremamente baixo $\left(\mathrm{n}=0,048 / \mathrm{m}^{2}\right)$ em relação às demais (Tabela 2). Para jovens, foram registrados após 10 meses do primeiro censo, 14 recrutas (10 oriundos de semente e com altura média de $74 \mathrm{~cm}$ e quatro de origem vegetativa e com altura média $78 \mathrm{~cm}$ ). Dos 14 jovens novos amostrados, oito foram registrados na população CM.

Cerne, Lavras, v. 18, n. 2, p. 265-273, abr./jun. 2012 
Tabela 2 - Número de sementes aparentemente viáveis, plântulas novas e percentual de estabelecimento de plântulas de Symphonia globulifera pântanos na RBPA (PORT, CM e ARI) e na RBU. Área amostral $=375 \mathrm{~m}^{2}$.

Table 2 - Number of apparently viable seeds, new seedlings and percentage of established seedlings of Symphonia globulifera in swamp forests in the RBPA (PORT, CM and ARI) and the RBU. Sample area $=375 \mathrm{~m}^{2}$.

\begin{tabular}{lccc}
\hline Pântanos & $\begin{array}{c}\text { Sementes } \\
\text { viáveis }\end{array}$ & $\begin{array}{c}\text { Plântulas } \\
\text { estabelecidas }\end{array}$ & $\begin{array}{c}\% \text { de } \\
\text { estabelecimento }\end{array}$ \\
\hline PORT & 18 & 2 & 11,1 \\
ARI & 98 & 6 & 6,1 \\
CM & 115 & 23 & 20 \\
RBU & 87 & 6 & 6,9 \\
\hline
\end{tabular}

\section{DISCUSSÃO}

A estrutura das populações de $S$. globulifera aqui estudadas mostrou-se dependente do regime hídrico do ambiente em que se encontravam. Os dados revelaram que não há uniformidade do terreno. Este é caracterizado por uma microtopografia de pequenos murunduns (ARAÚJO NETO et al., 1986) alternados com pequenas depressões e com erosão mais acentuada na RBU, o que explica os maiores valores de desvios padrão da profundidade média da lâmina d'agua. Pântanos mais profundos como PORT e ARI apresentaram populações com menor densidade de indivíduos e menor recrutamento. Vários estudos com espécies arbóreas tropicais têm demonstrado que, em geral, as populações são compostas em sua maioria por plântulas e jovens (CLARK; CLARK, 1992; NASCIMENTO; PROCTOR, 1997), com adultos representando menos de $10 \%$ da população. Esse padrão foi detectado nas populações CM e RBU, enquanto que as populações PORT e ARI apresentaram valores superiores a este padrão ( $24 \%$ e $27 \%$, respectivamente), com densidades de adultos muito inferiores que as encontradas para as de CM.

A ocorrência de reprodução tanto por via vegetativa quanto por via seminal é comum entre plantas que habitam ambientes alagados (CRAWFORD, 1992; SCARANO, 2006; SCARANO et al., 1997). A reprodução vegetativa pode ser uma alternativa para a espécie como estratégia potencial para emitir novos indivíduos em melhor época ou mesmo a de perpetuar a planta matriz (HARADA et al., 1997). É sabido que a heterogeneidade de habitats afeta o comportamento morfológico e demográfico das espécies (WIJESINGHE; HANDEL, 1994). A presença de clones pode ser uma das respostas à heterogeneidade de habitats, atuando, seja na otimização da exploração dos recursos seja na colonização.

Symphonia globulifera mostrou padrões de colonização pelas duas formas. Entretanto, a forma de reprodução por sementes foi predominante entre os adultos e jovens. Vale ressaltar que a população de clones adulta pode estar sendo subestimada, uma vez que não foi possível aferir como e quando os indivíduos se separam um dos outros se tornando independentes. Scarano et al. (1997), estudando uma área alagada também na Rebio Poço das Antas, encontraram cerca de $10 \%$ dos indivíduos $<1,0$ m de S. globulifera originados de reprodução vegetativa. Neste estudo, os valores encontrados de reprodução vegetativa foram $74 \%$ para plântulas (indivíduos $<50 \mathrm{~cm}$ ), $32 \%$ para jovens (indivíduos $\geq 50 \mathrm{~cm} \mathrm{e}<10 \mathrm{~cm}$ de DAP) e $22 \%$ para adultos (indivíduos $\geq 10 \mathrm{~cm} \mathrm{DAP)}$ ). Tanto as plântulas quanto os jovens de origem por sementes foram em média superiores em altura aos de origem vegetativa. As plântulas e jovens podem ter se beneficiado do fato da semente conter uma quantidade de reservas que os possibilitem um crescimento sem competição por nutrientes e livres de determinados conflitos aos quais os de origem vegetativa estão sujeitos. Segundo Hutchings (1986), esses conflitos podem ser: 1) competição entre os módulos conectados de um mesmo clone; 2) investimento e custo metabólico de diferentes tamanhos dos módulos produzidos; 3 ) habilidade das plântulas conectadas ganharem recursos da planta matriz.

$\mathrm{O}$ fato de a população PORT apresentar um menor número de novas plântulas oriundas de reprodução por sementes no segundo censo, coincidiu com as observações fenológicas (SANTOS, 1999), onde essa população, tal como a população ARI, apresentaram menor número de frutos maduros e queda precoce em relação a população $\mathrm{CM}$ e RBU. Aqui, novamente, os dados apontam para uma variação de comportamento fenológico entre as populações: as dos pântanos rasos (CM e RBU), produzindo mais sementes e também com o maior número de plântulas estabelecidas que as dos pântanos fundos (PORT e ARI). A baixa produção de frutos em relação ao número de flores produzidas de S. globulifera tem sido observada por outros pesquisadores na RBPA e RBU (LAPENTA; OLIVEIRA, 2003). Esse fato pode estar relacionado com à alta taxa de aborto em relação a produção de frutos observada em todos os estádios de

Cerne, Lavras, v. 18, n. 2, p. 265-273, abr./jun. 2012 
desenvolvimento do fruto. Essas taxas de aborto devem estar relacionadas a propriedades intrínsecas da espécie. Entretanto, é sabido que a inundação tende a aumentar a taxa de aborto de flores e frutos (CRAWLEY, 1986), principalmente em plantas menos tolerantes à inundação.

O sucesso de estabelecimento das plântulas parece estar relacionado à tolerância que estas podem apresentar ao regime de inundação, fato já observado para outras espécies arbóreas em florestas inundadas (SCHUPP, 1995; STEEG, 1994). S. globulifera mostra-se adaptada ao regime de inundação, com a dispersão de seus frutos ocorrendo na estação chuvosa, época em que o nível da água nos pântanos está no seu pico máximo. Algumas espécies são tolerantes a longos períodos de submersão (JUNK, 1989), porém não existem dados experimentais para Symphonia globulifera. Entretanto, o fato da germinação ocorrer com a semente ainda boiando e estas permanecerem em desenvolvimento por pelo menos três meses, conforme observado em nossos experimentos em laboratório, pode ser uma importante estratégia de colonização. Essa característica adiantaria o processo de fixação da plântula ao solo, possibilitando ganho de tempo e, consequentemente, altura suficiente para escapar da próxima cheia. Scarano et al. (2003) relatam a ocorrência dessa estratégia em Carapa guianensis Aubl. e relacionam essa habilidade da planta germinar e manter raizes e ramos vivos até a estação seca como importante para a ancoragem e o rápido estabelecimento quando da descida do nível d'água. Essa estratégia pode explicar o registro de jovens novos de $S$. globulifera, na segunda medição (maio/98). Assim, a dispersão das sementes de $S$. globulifera pode ser considerada principalmente hidrocórica. Entretanto, dados de campo revelaram que os morcegos também desempenham importante papel na dispersão dessas sementes.

\section{CONCLUSÕES}

A hipótese de que a estrutura das populações de $S$. globulifera nas matas de baixadas periodicamente inundadas é predominantemente composta por indivíduos de origem vegetativa face ao ambiente estressante e dinâmico que é o pântano foi parcialmente aceita, visto que houve predominância nos pântanos apenas de plântulas e jovens de origem vegetativa. A ocorrência de maior número de adultos (árvores) de $S$. globulifera oriundos de sementes sugere a existência de uma forte mortalidade diferenciada nas fases juvenis (plântulas e jovens), com maior mortalidade em indivíduos oriundos da reprodução vegetativa.

\section{AGRADECIMENTOS}

Aos pesquisadores Carlos Ruiz-Miranda, Dora M. Villela, Fábio R. Scarano e Jorge Assumpção pelas críticas ao manuscrito. A AMLD e ao IBAMA, por permitir o livre acesso à área de estudo. Ao PROBIO/ PRONABIO/BIRD/MMA pelo apoio financeiro e a FENORTE pela bolsa concedida ao primeiro autor. Ao CNPq pela bolsa de produtividade em pesquisa concedida à MTN.

\section{REFERÊNCIAS}

ALDRICH, P. R.; HAMRICK, J. L. Reproductive dominance of pasture trees in a fragmented tropical forest mosaic.

Science, New York, v. 281, p. 103-105, 1998.

ARAÚJO NETO, M. D.; FURLEY, P. A.; HARIDASAN, M.; JOHSON, C. E. The muruduns of the cerrado region of Central Brazil. Journal of Tropical Ecology, Cambridge, v. 2, p. 17-35, 1986.

BELLINGHAM, P. J.; SPARROW, A. D. Resprouting as a life history strategy in woody plant communities. Oikos, Copenhagen, v. 89, p. 409-416, 2000.

BITTRICH, V.; AMARAL, M. C. E. Pollination biology of Symphonia globulifera. Plant Systematics and Evolution, New York, v. 200, p. 101-110, 1996.

CARVALHO, F. A.; NASCIMENTO, M. T.; BRAGA, J. M. A.; RODRIGUES, P. J. F. P. Estrutura da comunidade arbórea da floresta atlântica de baixada periodicamente inundada na Rebio de Poço das Antas, RJ. Rodriguesia, Rio de Janeiro, v. 5, p. 503-518, 2006.

CLARK, D. A.; CLARK, D. B. Life history diversity of canopy and emergent trees in a neotropical rain forest. Ecological Monographs, Lawrence, v. 62, p. 315-344, 1992.

CORRÊA, B. S.; BERG, E. van den. Estudo da dinâmica da população de Xylopia brasiliensis Sprengel em relação a parâmetros populacionais e da comunidade em uma floresta de galeria em Itutinga, MG, Brasil. Cerne, Lavras, v. 8, p. 1-12, 2002.

Cerne, Lavras, v. 18, n. 2, p. 265-273, abr./jun. 2012 
CRAWFORD, R. M. M. Oxygen availability as an ecological limit to plant distribution. Advances in Ecological Research, London, v. 23, p. 93-185, 1992.

CRAWLEY, M. J. Life history and environment. In: Plant ecology. Oxford: Blackwell Scientific, 1986. p. 253290.

GILL, G. E.; FOWLER, R. T.; MORI, S. A. Pollination biology of Symphonia globulifera (Clusiaceae) in central French Guiana. Biotropica, Marburg, v. 30, p. 139-144, 1998.

HARADA, Y.; KAWANO, S.; IWASA, Y. Probability of clonal identidy: inferring the relative sucess of sexual versus clonal reproduction from spatial genetic patterns. Journal of Ecology, Oxford, v. 85, p. 591-600, 1997.

HOSSAERT-MCKEY, M.; VALERO, M.; MAGDA, D.; JARRY, M.; CUGUEN, J. E.; VERNET, P. The evolving genetic history of a population of Lathyrus sylvestris: evidence from temporal and genetic structure. Evolution, Lancaster, v. 50, p. 1808-1821, 1996.

HUTCHINGS, M. J. The structure of plant populations. In: CRAWLEY, M. J. (Ed.). Plant ecology. Oxford: Blackwell Science, 1986. p. 97-136.

JUNK, W. J. Flood tolerance and tree distribution in Central Amazonian floodplains. In: HOLM-NIELSEN, L. B.; NIELSEN, I.; BALSLEV, H. (Ed.). Tropical forests. New York: Academic, 1989. p. 65-72.

LAPENTA, M. J.; OLIVEIRA, P. P. Fruit exploitation by Golden Lion Tamarins (Leontopithecus rosalia) in the União Biological Reserve, Rio das Ostras, RJ, Brazil. Mammalia, Paris, v. 67, p. 41-46, 2003.

LIMA, H. C. de; PESSOA, S.; GUEDES-BRUNI, R.; MORAES, L. F.; GRANZOTTO, S. V.; IWAMOTO, S.; CIERO, J. Caracterização fisionômico-florística e mapeamento da vegetação da Reserva Biológica de poço das Antas, Silva Jardim, Rio de Janeiro, Brasil. Rodriguesia, Rio de Janeiro, v. 57, p. 369-390, 2006.

MARQUES, M. C. M.; JOLY, C. A. Estrutura e dinâmica de uma população de Calophyllum brasiliense Camb. em

Cerne, Lavras, v. 18, n. 2, p. 265-273, abr./jun. 2012 floresta higrófila do sudeste do Brasil. Revista Brasileira de Botânica, São Paulo, v. 23, p. 107-112, 2000.

NASCIMENTO, M. T.; CUNHA, C. N. da. Estrutura e composição florística de um cambarazal no Pantanal de Poconé, MT. Acta Botanica Brasilica, São Paulo, v. 3, p. 3-23, 1989.

NASCIMENTO, M. T.; PROCTOR, J. Population dynamics of five tree species in a monodominant Peltogyne forest and two other forest types on Maracá Island, Roraima, Brazil. Forest Ecology and Management, Amsterdam, v. 94, p. 115-128, 1997.

PAROLIN, P. Submerged in darkness: adaptations to prolonged submergence by woody species of the Amazonian Floodplains. Annals of Botany, London, v. 103, p. 359-376, 2009.

PERES, C. A. Costs and benefits of Territorial Defense in Golden Lion Tamarins. 1986. 134 f. Dissertação (Mestrado em Ecologia Animal) - University of Florida, Gainesville, 1986.

PRANCE, G. T.; SCHALLER, G. B. Preliminary study of some vegetation types of the Pantanal, Mato Grosso, Brazil. Brittonia, Bronx, v. 34, p. 228-251, 1982.

SANTOS, E. M. Estrutura populacional de Symphonia globulifera L. f. (Clusiaceae) em fragmentos de Mata Alagada nas reservas biológicas de Poço de Antas e Uniao. 1999. 67 f. Dissertação (Mestrado em Biociências) - Centro de Biociências e Biotecnologia, Campos dos Goytacazes, 1999.

SCARANO, F. R. Plant community structure and function in a swamp forest within the Atlantic rain forest complex: a synthesis. Rodriguesia, Rio de Janeiro, v. 57, p. 491-502, 2006.

SCARANO, F. R.; RIBEIRO, K. T.; MORAES, L. F. D.; LIMA, H. C. Plant establishment on flooded and unflooded patches of a freshwater swamp forest in southeastern Brazil. Journal of Tropical Ecology, Cambridge, v. 14, p. 793-803, 1997.

SCHUPP, E. W. Seed-seedling conflicts, habitat choice, and patterns of plant recruitment. American Journal of Botany, Columbus, v. 82, p. 399-409, 1995. 
SILANDER JUNIOR, J. A. Microevolucion and clone structure in Spartina patens. Science, Washington, v. 203, p. 658-660, 1985.

SOUZA, A. M.; CARVALHO, D.; VIEIRA, F. A.;

NASCIMENTO, L. H.; LIMA, D. C. Estrutura genética e espacial de populações naturais de Callophyllum brasiliense Camb. em Mata Atlântica de galeria. Cerne, Lavras, v. 13, n. 3, p. 239-247, 2007.

STEEG, H. ter. Flooding and drought tolerance in seeds and seedlings of two Mora species segregated along a soil hydrological gradient in tropical rain forest of Guyana. Oecologia, Berlin, v. 100, p. 356-367, 1994.

VELOSO, H. P.; RANGEL FILHO, A. L. R.; LIMA, J. C. A. Classificação da vegetação brasileira adaptada a um sistema universal. Rio de Janeiro: IBGE, 1991. 123 p.

WIJESINGHE, D. K.; HANDEL, S. N. Advantages of clonal growth in herogeneous habitats: an experiment with Potentilla simplex. Jounal of Ecology, Oxford, v. 82, p. 495502, 1994.

Cerne, Lavras, v. 18, n. 2, p. 265-273, abr./jun. 2012 